\title{
Automated inclusion and porosity analysis of metal additive manufacturing parts
}

Petrus Pistorius

Carnegie Mellon University, United States

Automated feature analysis allows rapid quantification of micron-sized pores and nonmetallic inclusions in additively manufactured metal parts. In this work, the focus is on parts produced by laser powder bed fusion.

The oxide concentration is high relative to wrought steel products: AM parts typically contain several hundreds of parts per million of oxygen. Automated analysis allows the origin, size, and severity of such inclusions to be assessed.

This presentation will give detail of some of the instrumental considerations, adding to our previous assessment [1]:

Choice of backscattered electron imaging conditions: The accelerating voltage has a strong effect on the visibility of inclusions. For oxide inclusions in aluminum alloys, the contrast is reversed if the interaction volume is larger than the inclusion, as shown by both Monte Carlo simulations and instrumental measurements. Brightness standards are essential to obtain consistent results. For superalloys and steels, the metal itself and aluminum tape work well; for aluminum, the alloy and graphite in the sample mount or carbon tape are suitable.

Choice of EDX conditions: Analysis at $10 \mathrm{kV}$ is preferred for inclusions in steels, since this limits the size of the interaction volume. At higher accelerating voltages, the peak count rates are distorted by differential absorption of characteristic X-rays by the surrounding steel matrix.

Control of these conditions ensures repeatable and rapid analyses, typically obtain several hundred analyses (size, shape and EDX composition) in twenty minutes, analyzing an area of some $9 \mathrm{~mm} 2$.

Additional morphological information is obtained by deep-etching the steel to expose nonmetallic inclusions. This allows imaging of the three-dimensional shape of the oxides. A suitable etchant is anhydrous methanol containing acetylacetone as chelant, plus tetramethylammonium chloride as supporting electrolyte. This etchant works well for steels and superalloys, but not for AlSi10Mg, since silicon is not dissolved by the chelant - though this etching method does work well to image the silicon cell structure in as-solidified parts.

\section{References}

[1] D. Tang, M.E. Ferreira and P.C. Pistorius: Automated inclusion microanalysis in steel by computerbased scanning electron microscopy: accelerating voltage, backscattered electron image quality, and analysis time. Microscopy and Microanalysis, vol. 23 (2017), pp. 1082-1090. DOI: $10.1017 / \mathrm{S} 1431927617012648$. 